\title{
LOS MISTERIOS QUE RODEAN LA ENFERMEDAD DE KAWASAKI: UNA REVISIÓN DE LA LITERATURA
}

\section{ARTÍCULO ORIGINAL}

ROSSI, Karoline ${ }^{1}$, MOREIRA, Danilo José Silva ${ }^{2}$, FONSECA, Juliana Brito da ${ }^{3}$, VASCONCELOS, Suzana dos Santos ${ }^{4}$, OLIVEIRA, Vinicius Faustino Lima de ${ }^{5}$, DIAS, Claudio Alberto Gellis de Mattos ${ }^{6}$, OLIVEIRA, Euzébio de ${ }^{7}$, DENDASCK, Carla Viana ${ }^{8}$, ARAÚJO, Maria Helena Mendonça de ${ }^{9}$, FECURY, Amanda Alves ${ }^{10}$

ROSSI, Karoline. Et al. Los misterios que rodean la enfermedad de Kawasaki: Una revisión de la literatura. Revista Científica Multidisciplinar Núcleo do Conhecimento. Año 06, Ed. 04, Vol. 05, pp. 52-64. Abril. ISSN: 2448-0959, Enlace de acceso: https://www.nucleodoconhecimento.com.br/salud/enfermedad-dekawasaki, DOI: 10.32749/nucleodoconhecimento.com.br/salud/enfermedad-dekawasaki

\section{RESUMEN}

La enfermedad de Kawasaki (DK) o síndrome del ganglio linfático mucocutáneo es una vasculitis sistémica, que afecta principalmente a niños menores de cinco años con ascendencia asiática, pero también puede llegar a otros grupos de edad, así

\footnotetext{
${ }^{1}$ Académico médico. Universidad Federal de Amapá (UNIFAP).

2 Un estudioso de la medicina. Universidad Federal de Amapá (UNIFAP).

${ }^{3}$ Académico médico. Universidad Federal de Amapá (UNIFAP).

${ }^{4}$ Académico médico. Universidad Federal de Amapá (UNIFAP).

${ }^{5}$ Un estudioso de la medicina. Universidad Federal de Amapá (UNIFAP).

${ }^{6}$ Biólogo, Doctor en Teoría e Investigación del Comportamiento, Profesor e investigador del Curso de Grado en Química del Instituto de Educación Básica, Técnica y Tecnológica de Amapá (IFAP).

${ }^{7}$ Biólogo, Doctor en Enfermedades Tropicales, Profesor e investigador del Curso de Educación Física de la Universidad Federal de Pará (UFPA).

${ }^{8}$ Teólogo, Doctor en Psicoanálisis, investigador del Centro de Investigación y Estudios Avanzados (CEPA).

${ }^{9}$ Doctor, Máster en Docencia y Ciencias de la Salud, Profesor e investigador del Curso Médico del Campus Macapá, Universidad Federal de Amapá (UNIFAP).

${ }^{10}$ Biomédico, Doctor en Enfermedades Tropicales, Profesor e investigador del Curso Médico del Campus Macapá, Universidad Federal de Amapá (UNIFAP).
}

RC: 82505

Disponível em: https://www.nucleodoconhecimento.com.br/salud/enfermedad-dekawasaki 
como a cualquier otra raza. El cuadro clínico de DK tiene tres etapas: etapa febril aguda, en la que la congestión coyuntural, la mucositis oral, el eritema, el descamación, la erupción polimórfica y la linfadenopatía laterocervical, aparecen como síntomas principales; la etapa subacute, que se produce al final de la fiebre, y conduce a la aparición de descamación de la piel en las extremidades, artritis, artralgia y trombocitosis y finalmente la etapa de convalecencia que surge cuando los síntomas son casi disipadores y continúa hasta su normalización. El tratamiento más utilizado se produce a partir de la administración de inmunoglobulina intravenosa, que para un mejor pronóstico de la patología debe iniciarse a tiempo.

Palabras clave: Síndrome del ganglio linfático mucocutáneo, Vasculitis, Arteritis, Fiebre, Kawasaki.

\section{INTRODUCCÍON}

La enfermedad de Kawasaki (DK) es una vasculitis sistémica que afecta a vasos pequeños y medianos y afecta principalmente a niños menores de 5 años, siendo una de las razones de la enfermedad cardíaca durante la infancia (HUANG et al., 2015; MAGALHÃES, 2008). Se desconoce la etiología de DK, es decir, los agentes causantes de esta enfermedad. Sin embargo, algunos aspectos clínicos y epidemiológicos proponen posibles razones infecciosas (FERRONATO et al., 2010).

DK es la segunda vasculitis más común en la edad pediátrica, afectando principalmente a niños menores de media década (90\%). Es poco común en niños menores de 6 meses y mayores de 8 años, que, sin embargo, son más propensos al desarrollo de aneurismas coronarios. A escala mundial, hay una variación en la ocurrencia, siendo Japón el país más afectado, con una incidencia anual de 110 a 150 casos por cada 100.000 niños menores de 5 años. Considerando el cálculo de las tasas de incidencia en cada 100.000 niños menores de 5 años, en los Estados Unidos de América (EE.UU.) DK es la causa más común de enfermedades cardíacas adquiridas en la infancia, que van de 9,1 a 32,5 casos, que ocurren con

RC: 82505

Disponível em: https://www.nucleodoconhecimento.com.br/salud/enfermedad-dekawasaki 
más frecuencia en los descendientes de islas asiáticas y del Pacífico $(32,5)$, intermedios en afroamericanos $(16,9)$ e hispanos $(11,1)$ y poco frecuentes en caucásicos (9,1) (ALMEIDA et al., 2010; CASTRO; URBANO; COSTA, 2009; RODRIGUES et al, 2017).

DK tiene una mayor prevalencia en la población asiática, con énfasis en el japonés, afectando principalmente a los niños. Hay un informe de una incidencia anual en Japón y Corea de hasta 100 casos por cada 100.000 habitantes, mientras que en la raza caucásica la ocurrencia es de entre 6 y 10 casos por cada 100.000 habitantes menores de media década. En América Latina, se estima que hay 3 casos por cada 100.000 habitantes, lo que evidencia una menor frecuencia de esta patología en poblaciones no asiáticas que no descienden de los asiáticos (SCARDINA et al., 2007). En cuanto a la tasa de mortalidad asociada a DK, es muy baja, ya que es inferior al 0,1\% (BARCA et al., 2019).

La incidencia de DK varía geográficamente en el mundo, siendo más frecuente en los descendientes japoneses, lo que sugiere una predisposición genética de la enfermedad, además de posibles agentes causatos que pueden estar vinculados a la ubicación geográfica, como las bacterias (CASTRO; URBANO; COSTA, 2009; RODRIGUES et al, 2017).

La variación estacional en la incidencia de DK es bien reconocida, pero las tasas cambian entre los países. En el Reino Unido, Australia y los Estados Unidos, hay un mayor número de casos en invierno y primavera. En China y Corea, en primavera y verano. En los Estados Unidos y el Reino Unido, el aumento de las tasas está más correlacionado con la variación de los períodos de lluvia que con la variación de la temperatura. En Brasil, un estudio realizado con 70 pacientes con DK mostró un registro más alto de la enfermedad coincidiendo con períodos de mayor incidencia de enfermedades infecciosas de mayo a junio (comienzo de la sequía) y noviembre y diciembre (lluvias tempranas) (MAGALHÃES; ALVES, 2017).

RC: 82505

Disponível em: https://www.nucleodoconhecimento.com.br/salud/enfermedad-dekawasaki 
Una persona con DK desarrolla características sintomáticas que conducen a la presentación de un cuadro clínico, que consiste en el conjunto de signos y síntomas manifestados por el paciente (KAYIRAN; DINDAR; GURAKAN, 2010). Por lo tanto, el cuadro clínico de DK se caracteriza por linfadenopatía cervical, fiebre, amigdalitis, hinchazón de las extremidades, erupción cutánea, picazón y descamación (ATIK, 2007; SCARDINA et al., 2007). Además, la DK puede causar inflamación generalizada, afectando a algunos órganos causando miocardiopatía inflamatoria, meningitis estéril, hepatitis, adenolinfito, pericarditis y angitis (CASTRO; URBANO; COSTA, 2009).

Castro, Urbano y Costa (2009) apuntan a una relación entre la vasculitis causada por DK y la implicación de algunos órganos, que consisten en la agrupación de tejidos responsables del mantenimiento del organismo.

DK fue reportado por primera vez en 1967 por el médico Japonés Tomisaku Kawasaki, quien lo definió como "síndrome del ganglio linfático mucocutáneo". Durante este período, se notificaron 50 casos en los años 1961 a 1967. En Japón, la ocurrencia es mayor, y la distribución global tiene su prevalencia variable (ALMEIDA et al., 2010; SCARDINA et al., 2007; MAGALHÃES; ALVES, 2017).

En el escenario actual, ya se han establecido algunos datos epidemiológicos sobre DK, lo que permite un análisis más preciso de los factores que intervienen en la difusión y propagación de enfermedades. Muchos estudios se han llevado a cabo con el fin de entender la fisiopatología de la enfermedad, que consiste en un conjunto de cambios anormales causados en el cuerpo (CASTRO; URBANO; COSTA, 2009).

En un estudio, se encontraron evidencias de DK y el avance de otras comorbilidades, lo que se refiere al hecho de que dos o más enfermedades están al mismo tiempo en un individuo, por lo que las complicaciones de DK serían la evolución desfavorable de esta asociación (SCARDINA et al., 2007).

RC: 82505

Disponível em: https://www.nucleodoconhecimento.com.br/salud/enfermedad-dekawasaki 
Para mejorar la atención y la calidad de vida del Paciente con DK, se desarrollaron tratamientos, que consisten en formas de cuidar a un paciente (CASTRO; URBANO; COSTA, 2009).

\section{METAS}

Detallar los posibles agentes causatos, la fisiopatología, los principales órganos y estructuras anatómicas afectadas por la enfermedad de Kawasaki (DK).

Verificar las principales características del cuadro clínico de un individuo para el diagnóstico, las principales complicaciones asociadas y los tratamientos disponibles para pacientes con DK.

\section{MÉTODO}

Se trata de una investigación bibliográfica llevada a cabo en las bases de datos Scielo, Biblioteca Digital Brasileña de Tesis y Disertaciones, LILACS y PubMed, utilizando palabras clave o sus asociaciones: enfermedad de Kawasaki-Kawasaki.

Los parámetros de inclusión utilizados en la preparación de las búsquedas fueron la disponibilidad completa en línea, el enfoque directo sobre la enfermedad de Kawasaki (DK) o en algún aspecto pertinente a esta enfermedad. En la base de datos Scielo había filtros de texto de las producciones escritas en inglés y portugués, en las otras bases de datos no se utilizó este criterio. Los parámetros de exclusión aplicados se duplicaron y se realizaron antes del año 2000.

Se hizo un análisis en las bases de datos mencionadas para enumerar los artículos relacionados con DK en la literatura. A partir de una lectura previa de los títulos y resúmenes de las producciones científicas encontradas, se descartaron estudios que no se correspondían con lo que se esperaba que constituyese esta revisión. Se leyeron los textos completos que pasaron por las etapas anteriores, seleccionando para esta revisión los que abordaban los aspectos pertinentes de la DK.

RC: 82505

Disponível em: https://www.nucleodoconhecimento.com.br/salud/enfermedad-dekawasaki 


\section{RESULTADOS}

\section{POSIBLES AGENTES CAUSATOS DE DK}

Todavía se desconoce la causa de la enfermedad de Kawasaki. Sin embargo, sus características clínicas y epidemiológicas alimentan la hipótesis de que un agente infeccioso es la causa desencadenante, que produce cambios en las células, tejidos y órganos de individuos con predisposición genética. Esta hipótesis es la más probable, pero todavía no tiene pruebas (CASTRO; URBANO; COSTA, 2009).

Una de las teorías es la del coronavirus NL-63, detectado por medio de una reacción en cadena de polimerasa (PCR) en fluidos de las vías respiratorias de 11 pacientes con DK y en sólo 1 de los 22 pacientes de control. Sin embargo, otros 5 estudios posteriores no encontraron coronavirus en muestras de tejido respiratorio o nasofarita de pacientes, lo que imposibilitó que este virus fuera la causa desencadenante (CASTRO; URBANO; COSTA, 2009).

Otra teoría es la de la estimulación inmunológica por superanógenos bacterianos como toxinas estafilococas y estreptocócicas. Una tercera teoría es inmunológica, que propone que la DK sea causada por una respuesta inmune aberrante en individuos genéticamente predispuestos (MAGALHÃES; ALVES, 2017).

\section{FISIOPATOLOGÍA DE DK}

Las alteraciones encontradas en DK, especialmente las histológicas, caracterizan una imagen de angiitis sistémica generalizada, afectando principalmente a vasos de calibre medio, especialmente las arterias coronarias. En varios órganos, se pueden observar cambios infames, como en el miocardio, en el pericardio, en los vasos sanguíneos, en las meninges, en el pulmón, en los ganglios linfáticos y en el hígado (CASTRO; URBANO; COSTA, 2009).

RC: 82505

Disponível em: https://www.nucleodoconhecimento.com.br/salud/enfermedad-dekawasaki 


\section{PRINCIPALES ÓRGANOS ANATÓMICOS $Y$ ESTRUCTURAS AFECTADAS POR DK}

La DK afecta a órganos y arterias, principalmente vasos de calibre medio de diversos sistemas del cuerpo humano, como cardiovasculares, pulmonares, gastrointestinales (el intestino y la vesícula biliar son los principales órganos afectados) y el sistema nervioso central (SNC). Sin embargo, cabe destacar la implicación que esta enfermedad tiene en el corazón, con la formación de aneurismas coronarios (CASTRO; URBANO; COSTA, 2009).

Otras arterias también pueden verse afectadas, tales como: aneurisma aórtico, con un mayor número de casos notificados que comprometen la aorta abdominal; aneurisma de la arteria axilar; aneurisma de la arteria braquiocéfala; aneurismas de arterias ilíacas, femorales; aneurisma de la arteria renal y obstrucción de las arterias oculares (ALVES et al., 2011).

\section{CARACTERÍSTICAS PRINCIPALES DEL CUADRO CLÍNICO DE UN INDIVIDUO PARA EL DIAGNÓSTICO DE DK}

La fiebre, que marca la fase aguda de la enfermedad, suele elevarse (por encima de $39^{\circ} \mathrm{C}$ ) y en picos, disminuyendo temporalmente en intensidad que dura aproximadamente siete a catorce días, y puede alcanzar tres y/o cuatro semanas si no hay un tratamiento adecuado. El día en que comienza la fiebre se considera el primer día de la enfermedad. Sin embargo, algunos pacientes tienen otras manifestaciones clásicas antes de la fiebre, tales como: conjuntivitis no purulenta; linfático cervical nodemegaly; eritemas y/o edemas en las manos y los pies; exanthetem escalatiniforme, morbiliforme o polimórfico; lengua framboesiforme, eritema y edema orofaríngeo, fisuras y eritema labial (CASTRO; URBANO; COSTA, 2009).

RC: 82505

Disponível em: https://www.nucleodoconhecimento.com.br/salud/enfermedad-dekawasaki 
Los cambios en las extremidades (manos y pies) con eritemas y/o edema también aparecen en la fase aguda, y el edema es muy doloroso y se asocia con eritema palmoplantar con vasculitis al alcance del dedo que después de un período de una a dos semanas, ya en fase de subaute, comienza a pelarse, comenzando por la región periungueal en el dedo guante (MAGALHÃES; ALVES, 2017).

La conjuntivitis bilateral no exudativa afecta especialmente a la conjuntiva bulbar en comparación con la conjuntiva palpebral y tarsal y es indolora. A veces se acompaña de iridocitocitis, una inflamación de la parte anterior del ojo, con resolución rápida y poco asociada con sensibilidad a la luz (CASTRO; URBANO; COSTA, 2009).

La erupción polimórfica es un conjunto de erupciones cutáneas que ocurren en los primeros días de la enfermedad, afectando principalmente el tronco y las extremidades, sin ampollas aparentes ni vesículas. Tienen presentación variable (erupción urticariforme, morbiliforme, maculopapular) o difusa, similar a la escarlatina (ALMEIDA et al., 2010).

Cambios en los labios y cavidad oral 9 de cada 10 casos en la fase aguda con hiperemia labial y orofaríngea intensa, fisuras, mucosa seca y sangrado de los labios. Las papilas de la lengua pueden llegar a ser prominentes y a veces se pueden observar ulceraciones orales con orofaringe exudato. Con respecto a la linfa cervical, otro criterio diagnóstico, el paciente debe presentar al menos un ganglio linfático con un diámetro mayor o igual a $1,5 \mathrm{~cm}$, comúnmente unilateral, doloroso y firme, en el triángulo cervical anterior (ALMEIDA et al., 2010; MAGALHÃES; ALVES, 2017).

\section{LAS PRINCIPALES COMPLICACIONES ASOCIADAS CON DK}

En pacientes con DK, se identifican tres tipos de vasculopatías en diferentes momentos de la evolución de la enfermedad: arteritis necrosante, arteritis subacute/crónica y vasculitis de proliferación miofibroblástica luminal. En la fase aguda de la enfermedad, destaca la arteritis necrotizante, que tiene su proceso

RC: 82505

Disponível em: https://www.nucleodoconhecimento.com.br/salud/enfermedad-dekawasaki 
patológico completo en las primeras dos semanas después del inicio de la condición febril, especialmente en las arterias coronarias, que pueden determinar la formación de aneurismas gigantes susceptibles al desarrollo de trombosis y rupturas. En la fase de subaute, se verifica que el proceso de vasculitis subacute comienza 2 semanas después de la aparición de fiebre, y puede afectar a todos los vasos sanguíneos, especialmente el segmento medio de las arterias musculares medias, como las arterias coronarias. En la fase crónica de la enfermedad, se produce mioproliferación fibroblástica luminal, asociada con vasculitis subacute crónica (MAGALHÃES; ALVES, 2017).

La complicación más grave de la enfermedad es la vasculitis coronaria, con cambios en las arterias coronarias, que afecta al $15-20 \%$ de los pacientes que no reciben tratamiento. Estas alteraciones incluyen aneurismas, ectasias y estenosis de las arterias coronarias, responsables del $2 \%$ de mortalidad (ALMEIDA et al., 2010).

En el sistema gastrointestinal, hay vómitos, diarrea, dolor abdominal e ileus paralítico. Al inicio de la fiebre, las manifestaciones más frecuentes son vómitos y diarrea y las menos comunes son ictericia, hepatomegalia dolorosa, pancreatitis y vesículas hidropic. En el sistema respiratorio, las manifestaciones más frecuentes son tos, neumonitis y cambios radiológicos. Rara vez ocurre rinorreia (MAGALHÃES; ALVES, 2017).

En el SNC, la enfermedad puede manifestarse con irritabilidad extrema en la fase aguda, además de meningitis aséptica, ataxia, parálisis facial y pérdida auditiva neurosensorial. También es destacable la presencia de ganglionitis y neuritis en nervios craneales y periféricos, endarteritis, periarteritis, coriomeningitis y leptomeningitis, además de atrofia, degeneración con pérdida de neuronas, gliosis marginal y subpendial y formación de nódulos gliales alrededor de degeneraciones neuronas (MAGALHÃES; ALVES, 2017).

Cambios conductuales como deterioro de la atención, deficiencia de aprendizaje, cambios emocionales (labilidad emocional, miedo y terror nocturno) y problemas de

RC: 82505

Disponível em: https://www.nucleodoconhecimento.com.br/salud/enfermedad-dekawasaki 
internalización (comportamiento ansioso, depresivo y agresivo) se han descrito en la literatura (ALVES et al., 2011).

\section{TRATAMIENTOS DISPONIBLES PARA LAS PERSONAS AFECTADAS POR DK}

La terapia para DK tiene dos propósitos diferentes dependiendo del escenario en el que se encuentre. En la fase aguda, tiene como objetivo reducir la respuesta inflamatoria en la pared arterial coronaria y prevenir la vasculitis y sus consecuencias (trombosis y aneurismas). En las fases subacúsica y crónica, el objetivo es prevenir la isquemia miocárdica y el infarto (CASTRO; URBANO; COSTA, 2009).

La inmunoglobulina intravenosa (IGEV) es el principal medicamento utilizado en DK y su mecanismo de acción sigue siendo desconocido. Se utiliza en la fase aguda, preferiblemente en los primeros 7 a 10 días del inicio de la afección (MAGALHÃES; ALVES, 2017).

El tratamiento consiste en la administración de inmunoglobulina en una sola dosis de $2 \mathrm{~g} / \mathrm{kg}$ durante un período variable de 10 a 12 horas junto con la administración de ácido acetilsalicílico (AAS) de 80 a 100 mg/kg/día, oralmente, 6/6 horas. La AAS de dosis altas tiene un efecto antiinflamatorio y, en dosis bajas, actúa como un inhibidor de la agregación plaquetaria, por lo que el tiempo de uso de AAS en dosis altas debe permanecer hasta que el niño sea afebrile. A continuación, la dosis se reduce a 3 a $5 \mathrm{mg} / \mathrm{kg} /$ día y se mantiene a esta dosis, mientras que el paciente presenta trombocitosis y/o cambios coronarios (MAGALLANES; ALVES, 2017; RODRIGUES et al., 2017).

Entre 1 y 2 de cada 10 pacientes con DK no responden a la primera dosis de IGEV, o con la permanencia de la condición febril o con la reaparición de la misma un día y medio después de la primera perfusión. Estos pacientes se consideran refractarios al tratamiento y son los que tienen mayor riesgo de cambios coronarios. En tales

RC: 82505

Disponível em: https://www.nucleodoconhecimento.com.br/salud/enfermedad-dekawasaki 
casos, se recomienda la administración de la segunda dosis de IGEV de $2 \mathrm{~g} / \mathrm{kg}$ en una sola dosis. Si la fiebre persiste durante 36 horas, se recomienda utilizar terapia de pulso con metilprednisolona a una dosis de $30 \mathrm{mg} / \mathrm{kg} /$ día (dosis máxima $1 \mathrm{~g} / \mathrm{día}$ ), infundida a 1 hora, 1 hora/día, durante 3 días (MAGALHÃES; ALVES, 2017).

En el tratamiento de la DK en las fases subacúsica y crónica, se utiliza aspirina en dosis bajas (3-5mg/ $\mathrm{kg} / \mathrm{día}$ ) en niños con aneurismas pequeños y medianos. En estas fases, el tratamiento tiene como objetivo prevenir la trombosis (activación plaquetaria) y la estelasis del vaso. También se utilizan otros agentes antiplaquetarios (clopidogrel, ticlopidina, dipyridamol) y, asociados con la aspirina, se presentan como más concretos en el bloqueo de la agregación plaquetaria (CASTRO; URBANO; COSTA, 2009).

\section{DIscusíon}

\section{CORRELACIÓN ENTRE LOS POSIBLES CAUSANTES Y EL DESARROLLO DE DK}

Hasta la fecha, el origen de DK no ha sido identificado, pero hay algunos agentes, que se cree que están relacionados con el desarrollo de la patología. Estos son Staphylococcus aureus, estreptococos, vírus influenza, morbilivírus, paramixovírus, bunyavírus. El adenovirus y un nuevo coronavirus humano llamado coronavirus new haven (Nco-NH) también pueden estar asociados con DK, ya que ya han sido identificados en secreciones de vías respiratorias de pacientes con la enfermedad. Estos virus y bacterias podrían actuar sobre el desarrollo de DK, de modo que conducirían a datos causados por la enfermedad, como aneurismas aórticos (BARCA et al., 2019; CASTRO; URBANO; COSTA, 2009).

Hay una hipótesis que asocia la existencia de superanógenos bacterianos con el desarrollo de DK. Los estudios que analizan esta sugerencia suponen que el

RC: 82505

Disponível em: https://www.nucleodoconhecimento.com.br/salud/enfermedad-dekawasaki 
superanógeno puede unirse a la célula que presenta antígenos, a través del complejo de histocompatibilidad mayor de clase II (MHC de clase II) y el receptor de linfocitos $\mathrm{T}$ en sitios de unión diferentes de los de antígenos comunes. Esto promovería una respuesta inmunológica con la formación y liberación de citoquinas proinflamatorias en concentraciones excesivas, contribuyendo a la evolución de un proceso inflamatorio característico de DK (CASTRO; URBANO; COSTA, 2009).

Algunos indicios conducen a la creyción de que una respuesta oligoclonal lgA ocurre en DK agudo. Un estudio realizado por Castro, Urbano y Costa (2009) realizó el análisis de la cadena alfa pesada de inmunoglobulinas recogidas de una gran cantidad de leucocitos acumulados en un sitio de respuesta inflamatoria de la pared arterial de pacientes con DK, que mostró que se detectaron anticuerpos específicos de inmunoglobulina $A(\lg A)$ en la muestra. Esto evidencia la existencia de una respuesta inmune relacionada con DK (CASTRO; URBANO; COSTA, 2009).

La hipótesis de que DK tiene predisposición genética se ve reforzada por la alta incidencia entre los asiáticos y sus rummies. Un estudio mostró 67 genes relacionados con el desarrollo de la patología, que implicaban la participación en el endotelio, el metabolismo lipídico, la activación de mecanismos inmunológicos y el reclutamiento plaquetario, apoyando la idea de que existe una relación genética para el desarrollo de DK (CASTRO; URBANO; COSTA, 2009; SOTELO; GONZÁLEZ, 2007).

\section{FISIOPATOLOGÍA DE DK}

La vasculitis comienza con la aparición de hinchazón e inflamación, que pueden ocurrir durante un período variable. Los neutrófilos están presentes inicialmente, y poco después predominan los linfocitos T citotóxicos CD8+ y la inmunoglobulina $\lg A$ (CASTRO; URBANO; COSTA, 2009).

En las arterias coronarias hay cambios inflamatorios con la presencia de edema y necrosis de células musculares. Con esta pérdida de integridad estructural existe la

RC: 82505

Disponível em: https://www.nucleodoconhecimento.com.br/salud/enfermedad-dekawasaki 
formación de aneurismas. Además, los fibroblastos proliferan y luego remodelan, lo que conduce a la posible presencia de sustesis, calcificaciones y trombosis (CASTRO; URBANO; COSTA, 2009; SOTELO; GONZÁLEZ, 2007).

Es importante tener en cuenta que el factor inflamatorio 1 que se encuentra en los aloinjertos se incrementa bruscamente en los tejidos arteriales eshimnóticos de DK. Este factor inflamatorio 1 del aloinjerto une la respuesta del interferón tipo I hasta la activación de macrófagos específicos y linfocitos $\mathrm{T}$, que asume la probable importancia de la interferencia de estas células en la arteritis por DK (ROWLEY et al., 2017).

\section{CÓMO LOS ÓRGANOS PRINCIPALES Y LAS ESTRUCTURAS ANATÓMICAS SE VEN AFECTADOS POR DK}

La DK afecta a órganos y arterias, principalmente vasos de tamaño mediano, como los del corazón, específicamente las arterias coronarias, con la formación de aneurismas. La enfermedad también puede afectar el sistema respiratorio (pulmón), a través de vasculitis, el sistema gastrointestinal, principalmente el intestino y la vesícula biliar, y el sistema nervioso (CASTRO; URBANO; COSTA, 2009).

El sistema linfático puede verse afectado por la DK, ya que uno de los síntomas de la DK es la hinchazón de los ganglios linfáticos cervicales. El sistema cutáneo está dañado por eritema cutáneo, seguido de edemas. En los ojos, puede producirse hiperemia de coyuntura no suputiva y las membranas mucosas de la cavidad oral pueden verse afectadas por mucositis oral y lesiones en los labios (SOTELO; GONZÁLEZ, 2007).

\section{LAS PRINCIPALES CARACTERÍSTICAS DEL CUADRO CLÍNICO DE UN INDIVIDUO CON DK Y CÓMO LE AFECTAN}

El curso clínico de DK se puede dividir en tres etapas clínicas: aguda, subacute y convalecencia. La etapa febril aguda generalmente dura de 7 a 14 días,

RC: 82505

Disponível em: https://www.nucleodoconhecimento.com.br/salud/enfermedad-dekawasaki 
caracterizada por congestión coyuntural, mucositis oral, eritema, erupción descamación y polimórfica y linfadenopatía. La etapa de subaute es el período entre el final de la fiebre y el día 25 de la enfermedad. En esta etapa, los pacientes presentan descamación de la piel en las extremidades, artritis, artralgia y trombocitosis. La etapa de convalecencia comienza cuando los síntomas clínicos comienzan a desaparecer y continúa hasta el establecimiento normal de VHS (tasa de presión arterial roja), que generalmente se observa de 6 a 8 semanas después de la aparición de los síntomas. Tales etapas afectan la vida del paciente, ya que le impiden realizar sus actividades diarias, que se debe principalmente a lesiones cutáneas y sintomatología (ALMEIDA, 2017; SCARDINA et al., 2007).

La afección sugerente de la patología se caracteriza por fiebre, aumento de los ganglios linfáticos cervicales y amigdalitis, seguido de eritema cutáneo generalizado, picazón y edema de las extremidades y después de unos días de descamación. Las pruebas de laboratorio muestran una fase inflamatoria activa con alta tasa de sedimentación, proteína $\mathrm{C}$ reactiva, leucocitosis y un mayor número de plaquetas, lo que conduce al letargo del individuo afectado, ya que la inflamación puede causar dolor intenso (ALMEIDA, 2018; ATIK; FORONDA; BUSTAMANTE, 2003; FERRONATO et al., 2010; ROSSI et al., 2015).

También puede surgir hiperemia de coyuntura no suputiva; erupción macularpapular; lesiones en los labios y cavidad oral; cambios en la piel de las extremidades, además del soplo precordial, dificultad para respirar, dolor abdominal, disuria, hepatomegalia, artralgia, rigidez del cuello y convulsiones. Estos síntomas impiden al paciente ver y moverse con normalidad, ya que los ojos están dañados y el dolor causa dificultad, para realizar movimientos (CASTRO; URBANO; COSTA, 2009; SOTELO; GONZÁLEZ, 2007).

DK afecta a varios pacientes con vasculitis en varios órganos, como se ha dicho anteriormente, el pulmón, intestino, vesícula biliar, sistema nervioso central, son ejemplo, lo que conduce a la aparición de síntomas como fatiga, pérdida de peso y 
dolor en las articulaciones y músculos, pero el daño cardíaco es el más significativo, con la formación de aneurismas coronarios, que conducen a la angina de pecho (CASTRO; URBANO; COSTA, 2009; PRINTZ, 2011).

Otro síntoma es la irritación, que aparece en niños con la enfermedad, y las quejas gastrointestinales, incluyendo diarrea, vómitos y dolor abdominal, ocurren en aproximadamente un tercio de los pacientes, lo que explica el hecho de que los niños con DK están más irritados que los demás (ALMEIDA, 2018; KAYIRAN; DINDAR; GURAKAN, 2010).

El absceso y el edema retrofaríngeo generalmente se asocian con una afección mortal si no se tratan correctamente. Los otorrinolaringólogos pueden tratarlo con drenaje quirúrgico del absceso, evitando el bloqueo de las vías respiratorias (KIM; KWON, 2016; XIE et al., 2018).

La mucositis oral está representada por el eritema difuso de la membrana mucosa, el enrojecimiento en los labios y la lengua, y la hipertrofia de las papilas linguales con posterior desarrollo de la lengua de fresa, y generalmente ocurre en la etapa aguda de la enfermedad y la convalecencia, como consecuencia del tratamiento farmacológico, e impide que el paciente se alimente adecuadamente debido a las lesiones, afectando a su nutrición adecuada (SCARDINA et al., 2007).

\section{EI DIAGNÓSTICO DE DK}

DK representa un desafío que debe diagnosticarse a tiempo, ya que es un trastorno reumatológico inusual que requiere una intervención específica para evitar secuelas graves o fatales (ALMEIDA et al., 2010).

La clínica de la enfermedad no está clara, siendo común el diagnóstico inicial de otras enfermedades, como escarlatina, exantesis virales o afecciones alérgicas, debido a los síntomas iniciales de la enfermedad. Incluso con el cumplimiento completo de los criterios, es frecuente retrasar el diagnóstico correcto, lo que

RC: 82505

Disponível em: https://www.nucleodoconhecimento.com.br/salud/enfermedad-dekawasaki 
conduce a un retraso en el inicio del tratamiento adecuado, favoreciendo la aparición de complicaciones, que pueden agravar rápidamente la condición clínica del paciente. Además, existen formas incompletas o atípicas, que dificultan aún más el diagnóstico. El diagnóstico precoz de la enfermedad es importante, ya que el tratamiento en la fase aguda reduce el proceso inflamatorio en las arterias coronarias y previene la trombosis, reduciendo las posibilidades de mortalidad (FERRONATO et al., 2010).

El diagnóstico clínico oportuno es esencial para establecer este tratamiento temprano y evitar lesiones coronarias. Este tipo de lesión puede causar isquemia o infarto de miocardio, que es extremadamente grave (KAYIRAN; DINDAR; GURAKAN, 2010; SOTELO; GONZÁLEZ, 2007).

Los criterios diagnósticos de la enfermedad clásica de DK son la fiebre con cinco días o más, además de al menos otros cuatro criterios, que son: eritema palmoplantar con o sin edema doloroso o descamación periuniguense; Exatheme polimórfico; hiperemia conjuntival bulbar en ambos lados, no exudativa; Cambios en los labios y mucosa oral; Linfadenopatía cervical - diámetro superior a $1,5 \mathrm{~cm}$, duro e indoloro en palpación. El diagnóstico de DK incompleto puede establecerse en presencia de tres o más de los siguientes criterios: Albúmina $\leq 3 \mathrm{~g} / \mathrm{dL}$; Anemia; Elevación de alanina aminotransferasa; Plaquetas después de siete días $\geq$ $450.000 / \mathrm{mm}^{3}$; Leucocitos $\geq 15.000 / \mathrm{mm}^{3}$; Orina $\geq 10$ leucocitos (CASTRO; URBANO; COSTA, 2009; RODRIGUES et al., 2017; YAP; LIN; WANGUI, 2012).

Las principales secuelas de la enfermedad están relacionadas con el sistema cardíaco, por lo tanto, las imágenes cardíacas por ecocardiografía son una parte relevante de la evaluación de pacientes con DK sospechoso. Para casos sin complicaciones, se recomienda ecocardiografía en el diagnóstico, de 6 a 8 semanas después de la aparición de la enfermedad (ATIK et al., 2017; KAYIRAN; DINDAR; GURAKAN, 2010)

RC: 82505

Disponível em: https://www.nucleodoconhecimento.com.br/salud/enfermedad-dekawasaki 
Las alteraciones más frecuentes son el recuento sanguíneo con leucocitosis y neutrofilia, además de aumentar los valores de las pruebas de fase aguda, como la velocidad de la hemosedimentación y la proteína C reactiva. La enfermedad a menudo se diagnostica sólo después de la afectación de las arterias coronarias (FERRONATO et al., 2010).

Un estudio de Kentsis et al. (2013) constató que se detectaron marcadores de filamina C y meprina A tanto en suero como en orina de los dos grupos independientes de pacientes con DK participantes. El mismo estudio considera que el uso de estos marcadores para realizar el diagnóstico de DK es más eficiente que el uso de marcadores utilizados actualmente (KENTSIS et al., 2013).

\section{EXÁMENES DE IMAGEN Y COMPLEMENTARIOS}

El ecocardiograma es una de las principales pruebas utilizadas para el diagnóstico de DK, ya que no es invasivo, tiene una alta sensibilidad y especificidad para analizar y detectar anomalías en las arterias coronarias, evaluar el miocardio y verificar la presencia de pericarditis. En este examen, pueden aparecer algunos hallazgos que indican arteritis coronaria, que precede a la formación de aneurismas, como el brillo perivascular, la ectasia o el estrechamiento de las arterias coronarias. En el análisis de las arterias coronarias, debe evaluarse el diámetro interno del vaso; el número, la ubicación y el tipo (ya sean sacculares o fusiformes) de los aneurismas; y la presencia o no de trombo intraluminal y sustesis. En la fase aguda de la enfermedad, algunos hallazgos frecuentes en ecocardiogramas son: reducción de la contractilidad ventricle izquierda, regurgitación de la válvula mitral y derrame pericárdico (CASTRO; URBANO; COSTA, 2009; MAGALHÃES; ALVES, 2017; RODRIGUES et al., 2017).

Otras pruebas que se pueden realizar, en función de la disponibilidad, gravedad, ventajas e indicaciones que varían según el caso. Por ejemplo, resonancia

RC: 82505

Disponível em: https://www.nucleodoconhecimento.com.br/salud/enfermedad-dekawasaki 
magnética (RNM), angioresonance, radiografía de tórax, tomografía computarizada de alta resolución, angiografía (CASTRO; URBANO; COSTA, 2009).

Últimamente, la tomografía computarizada de alta resolución ha sido más indicada, especialmente en comparación con la RNM, ya que su ejecución es más rápida y fácil de interpretar, mientras que la RNM requiere más tiempo para que los niños recolecten anestésicos (CASTRO; URBANO; COSTA, 2009).

Últimamente, la tomografía computarizada de alta resolución se ha indicado más, especialmente en comparación con la RMN, ya que su ejecución es más rápida y fácil de interpretar, mientras que la RMN requiere un tiempo anestésico más largo de los niños para la recolección (CASTRO; URBANO; COSTA, 2009).

Se descubren radiografías torácicas, que generalmente no presentan alteraciones, en aproximadamente el $15 \%$ de los casos se descubren anomalías, tales como: patrón de infiltración reticulogranular o peribronquial, acumulación de demasiado líquido en el espacio entre las pleuras viscerales y parietales o colapso del pulmón. Aparecen después del décimo día de la enfermedad y son capaces de comprobar en radiografías de 10 a 50 días después del inicio de la afección (CASTRO; URBANO; COSTA, 2009).

En las pruebas de laboratorio, los hallazgos no son específicos de la DK, sino más bien una indicación de un proceso inflamatorio alto y es posible encontrar, al comienzo de la fase aguda, un aumento en la velocidad de hemosedimentación (VHS), proteína C reactiva (PCR) y tasas de alfaglicoproteína ácida. En el análisis de sangre es posible comprobar una cantidad anormalmente alta de neutrófilos, llamados leucocitosis con neutrofilia, y puede ir acompañada de aumento de murciélagos o eosinofilia. Además, la cantidad de plaquetas suele ser normal en la fase aguda, sin embargo, es posible que en algunos casos haya trombocitopenia, que se asocia con el desarrollo de enfermedad coronaria grave e infarto de miocardio (MAGALHÃES; ALVES, 2017).

RC: 82505

Disponível em: https://www.nucleodoconhecimento.com.br/salud/enfermedad-dekawasaki 


\section{ANÁLISIS DE LAS PRINCIPALES COMPLICACIONES ASOCIADAS CON DK}

La complicación más grave de la enfermedad es la vasculitis coronaria, que afecta al $15-20 \%$ de los pacientes no tratados, lo que puede conducir a aneurismas, ectasias y sustesis de las arterias coronarias, que representan un $2 \%$ de mortalidad. Otras complicaciones de la DK se han descrito como aneurismas en otras arterias como la aorta; la aorta abdominal; arteria axilar; arteria braquiocéfala; arterias ilíacas, arterias femorales y arterias renales, lo que indica cómo DK puede afectar enormemente al cuerpo humano, y por lo tanto ser extremadamente peligroso (ALVES, et al., 2011; CHBEIR et al., 2018; MUTLUER; ÇELIKER, 2019; PRINTZ, 2011; XIE et al., 2018).

Algunos pacientes con DK son susceptibles a la dilatación coronaria progresiva. Dicha dilatación de las arterias coronarias se produce durante la fase aguda y puede proceder a la fase de subaute, lo que agrava la condición clínica de varios pacientes (BRIEDÉ et al., 2015; 2015; LIU et al., 2017).

Pueden ocurrir complicaciones gastrointestinales en DK como obstrucción intestinal, edema de colon, isquemia intestinal, pseudo-obstrucción intestinal y abdomen agudo, que afecta al paciente de manera alarmante, ya que estas complicaciones pueden conducir a sepsis e incluso a la muerte. Además, las alteraciones oftalmológicas asociadas con la DK pueden incluir uveítis, iridocitocitis, hemorragia conjuntival, neuritis óptica, amaurosis y obstrucción de la arteria ocular, que causa secuelas irreparables varias veces. DK también se manifiesta como vasculitis necrosante, lo que conduce a la pérdida de tejido afectado (ALVES, et al., 2011).

Las complicaciones neurológicas debidas a la lesión cns pueden ser las siguientes: meningoencefalitis, recolección subdural, hipoperfusión cerebral, isquemia, infarto cerebral, convulsiones, emiplejia, confusión mental, letargo e incluso coma o infarto cerebral. Tales complicaciones pueden volverse irreversibles, dañando así para siempre la vida del paciente (ALVES, et al., 2011).

RC: 82505

Disponível em: https://www.nucleodoconhecimento.com.br/salud/enfermedad-dekawasaki 
El riesgo de complicaciones tardías de DK sugiere la necesidad de un seguimiento a largo plazo además de la infancia (GIACCHI et al., 2014).

\section{TRATAMIENTOS DISPONIBLES PARA LAS PERSONAS AFECTADAS POR DK}

El tratamiento más indicado es la aplicación de IGIV (Inmunoglobulina Intravenosa) en una sola dosis de $2 \mathrm{~g} / \mathrm{kg}$ por perfusión de 12 horas. Según la evolución del quaro clínico del paciente, la dosis de IGIV puede repetirse o se pueden añadir corticosteroides, especialmente en casos refractarios. Se recomiendan otros medicamentos, como ciclofosfamida, ciclosporina y ulinastatina. Además, se recomienda un producto basado en anticuerpos monoclonales contra el factor alfa de necrosis tumoral, así como un anticuerpo monoclonal, conocido como Abciximab, y que puede tratar los aneurismas más rápidamente (ATIK, 2007; FERRONATO et al., 1010; KAYIRAN; DINDAR; GURAKAN, 2010; PHILIP et al., 2017; SOTELO; GONZÁLEZ, 2007).

Aproximadamente entre el $10 \%$ y el $15 \%$ de los pacientes con KD no muestran mejoría con el tratamiento IVIG, que es el tratamiento estándar (MEHNDIRATTA et al., 2014). En pacientes pediátricos con DK, altas dosis de ácido acetilsalicílico (AAS) proporcionan acción antiinflamatoria, mientras que las dosis bajas tienen un efecto inhibitorio de la agregación plaquetaria. Así, en la fase aguda de la enfermedad, se utiliza AAS $80-100 \mathrm{mg} / \mathrm{kg} / \mathrm{d}$ ía, que se divide en cuatro ingestiónes a lo largo del día, con el fin de intensificar el efecto antiinflamatorio de la IGIV. Mientras que en algunos hospitales la dosis de AAS se reduce después de 2-3 días en ausencia de fiebre, en otros centros de salud el tratamiento persiste hasta 2 semanas. Además, los niños con anomalías cardíacas tienen una receta para auditivos indefinidamente (ATIK, 2007; CASTRO; URBANO; COSTA, 2009; MEHNDIRATTA et al., 2014).

RC: 82505

Disponível em: https://www.nucleodoconhecimento.com.br/salud/enfermedad-dekawasaki 
También se utilizan medicamentos inmunosupresores como la ciclofosfamida, junto con la prednisona o la metilprednisolona, para mejorar algunos síntomas, como la fiebre, pero estos medicamentos no tienen un efecto terapéutico sobre las anomalías cardíacas (CASTRO; URBANO; COSTA, 2009; PHILIP et. al., 2017).

En la fase aguda de la enfermedad de DK, existe un mayor riesgo quirúrgico porque, en presencia de inflamación, la anastomosis con arteria coronaria inflamada corre el riesgo de obstrucción. El enfoque quirúrgico debe limitarse a pacientes con aneurismas gigantes o procesos isquémicos después de la fase aguda (ATIK, 2007; SOTELO; GONZÁLEZ, 2007).

La revascularización quirúrgica de lesiones coronarias secundarias a la DK es relativamente poco frecuente. De acuerdo con las directrices actuales, la revascularización arterial completa debe utilizarse en pacientes jóvenes con pequeñas comorbilidades con el fin de obtener una buena permeabilidad al injerto a largo plazo (BARCA et. al., 2019).

Además de la cirugía de revascularización miocárdica, que ha sido el tratamiento de elección para complicaciones coronarias trombóticas de DK, La intervención coronaria percutánea $(\mathrm{PCl})$ con stents regulares 0 percutáneos con politetrafluoroetileno (PTFE), implantación de bobinas, revascularización coronaria transluminal percutánea (CRT) con agentes trombolíticos en la arteria relacionada con el infarto y ablación rotacional coronaria percutánea (PTCRA), se utilizan en pacientes afectados por la enfermedad (MUTLUER; ÇELIKER, 2019).

En cuanto al manejo anestésico, hay poca información descrita, sin embargo, la revascularización miocárdica con anestesia realizada por gases halogenados presenta menos daño, mostrando así una mejor recuperación postoperatoria de la función miocárdica en comparación con los anestésicos intravenosos. Así, favorece la inhibición de la respuesta neuroendocrina al estrés, y también prepara al paciente para una extubación temprana en el postoperatorio, lo que ayuda en la prevención

RC: 82505

Disponível em: https://www.nucleodoconhecimento.com.br/salud/enfermedad-dekawasaki 
de posibles complicaciones derivadas de la intubación (MARTíNEZ; MÉNDEZ, 2013).

\section{CONCLUSIÓN}

La aparición de DK es variable, estando más presente en la región de Asia, especialmente en niños menores de 5 años. En América Latina la incidencia es menos frecuente y la mortalidad de esta patología es baja.

El agente etológico de DK no ha sido identificado, pero hay algunos patógenos que pueden estar relacionados con esta enfermedad. Hay evidencia de que esta enfermedad tiene una relación genética, esto se encontró debido al aumento de la incidencia entre los asiáticos y entre los familiares de los pacientes afectados por DK, además, también hay algunos genes que están asociados con esta patología.

Hay alteraciones asociadas con la DK como inflamaciones sistémicas, que afectan a ciertos órganos causados por angiitis, meningitis estéril, neumonitis, adenofinita y hepatitis. La DK afecta a órganos y arterias, principalmente vasos de calibre medio, como los del corazón, por lo que conduce a la formación de aneurismas, pueden afectar los ojos, las membranas mucosas de la cavidad oral, el pulmón, el intestino, la vesícula biliar y el sistema nervioso, el sistema linfático, el sistema cutáneo y las lesiones pueden ocurrir en los labios.

El cuadro clínico de DK tiene tres etapas. La etapa febril aguda se caracteriza por congestión coyuntural, mucositis oral, eritema, descamación, erupción polimórfica y linfadenopatía laterocervical. La etapa subacute ocurre al final de la fiebre, los pacientes tienen descamación de la piel en las extremidades, artritis, artralgia y trombocitosis. La etapa de convalecencia surge cuando los síntomas casi desaparecen y continúan hasta su normalización.

El retraso en el diagnóstico correcto de DK es bastante frecuente, lo que puede conducir a un retraso en el inicio del tratamiento, lo que puede causar 
complicaciones para empeorar la condición clínica. El diagnóstico clínico temprano es extremadamente importante para establecer un tratamiento temprano y evitar lesiones de las arterias coronarias.

El tratamiento de la DK se basa en la evolución del cuadro clínico del paciente. La terapia más utilizada es la aplicación de IGIV, si se producen cambios en la clínica, se puede repetir la dosis de IGIV o se pueden añadir corticosteroides. Se pueden indicar otros medicamentos, como ciclofosfamida, ciclosporina y ulinastatina. También se recomienda un producto basado en anticuerpos monoclonales Infliximab, inmunosupresores como ciclofosfamida o ciclosporina y prednisona o metillprednisolona se pueden utilizar. El tratamiento quirúrgico se realiza principalmente en la fase aguda de la DK, pero debe limitarse a pacientes con aneurismas gigantes o procesos isquémicos después de la fase aguda de la enfermedad.

\section{REFERENCIAS}

ALMEIDA, F. C. Avaliação das manifestações clínicas e achados laboratoriais em 301 pacientes com doença de Kawasaki: acompanhamento de dez anos. Tese (Doutorado em Ciências Médicas). Faculdade de Medicina, Universidade de Brasília, Brasília. 2018.

ALMEIDA, M. A. A. L. S. Avaliação da calprotectina e dos anticorpos anticitoplasma de neutrófilos como marcadores de inflamação e autoimunidade nas diferentes fases da doença de Kawasaki. Dissertação (Mestrado em Ciências Farmacêuticas). Faculdade de Ciências da Saúde, Universidade de Brasília, Brasília. 2017.

ALMEIDA, R. G. et al. Perfil da doença de Kawasaki em crianças encaminhadas para dois serviços de reumatologia pediátrica do Rio de Janeiro, Brasil. Revista Brasileira de Reumatologia, v. 50, n. 5, p. 529-538, set./out. 2010.

RC: 82505

Disponível em: https://www.nucleodoconhecimento.com.br/salud/enfermedad-dekawasaki 
ALVES, N. R. M. et al. Estudo prospectivo das complicações da Doença de Kawasaki: análise de 115 casos. Revista da Associação Médica Brasileira, v. 57, n. 3, p. 299-305, mar. 2011

ATIK, E. Doença de Kawasaki: Regressão de Aneurismas Gigantes das Artérias Coronárias com Obstrução Tardia Posterior Kawasaki Disease: Giant Coronary Arteries Aneurysms Regression and Later Stenosis. Arquivos brasileiros de Cardiologia, v. 88, n. 1, p. 22-23, 2007.

ATIK, E.; FORONDA, A.; BUSTAMANTE, L. N. P. Kawasaki Disease. Involution of Giant Coronary Aneurysms After Prolonged Anti-inflammatory Treatment. Arquivos brasileiros de Cardiologia, v. 81, n. 3, p. 265-72, set. 2003.

ATIK, E. et al. Caso 6/2017 - Extenso Aneurisma Gigante de Artéria Coronária Esquerda por Vasculite de Kawasaki em Homem Assintomático com 48 Anos de Idade. Arquivos brasileiros de Cardiologia, v. 109, n. 5, p. 489-490, nov. 2017.

BARCA, L. V. et al. Ligature of the Left Main Coronary Artery after Surgery in Kawasaki Disease: Case Report. Braz J Cardiovasc Surg, v. 34, n. 1, p. 111-113, jan./fev., 2019.

BRIEDÉ, S. et al. Hamoen M, Oosterveld M JS, Breur JMPJ. Langetermijneffecten van de ziekte van Kawasaki. Ned tijdschr geneeskd, v. 154, n. 2121, p. 1-6, out. 2015.

CASTRO, P. A.; URBANO, L. M. F.; COSTA, I. M. C. Doença de Kawasaki. Anais Brasileiros de Dermatologia, v. 84, n. 4, p. 317-331, mai. 2009.

CHBEIR, D. et al. Kawasaki disease: abnormal initial echocardiogram is associated with resistance to IV Ig and development of coronary artery lesions. Pediatric Rheumatology Onlinw Journal, v. 6, n. 48, p. 1-10, jul. 2018.

RC: 82505

Disponível em: https://www.nucleodoconhecimento.com.br/salud/enfermedad-dekawasaki 
FERRONATO, A. E. et al. Doença de Kawasaki: experiência clínica em hospital universitário. Revista Paulista de Pediatria, v. 28, n. 2, p. 148-154, jun. 2010.

GIACCHI, V. et al. Avaliação do espessamento íntimo da artéria coronária em pacientes com diagnóstico prévio de doença de Kawasaki por meio de ecocardiografia transtorácica de alta resolução: nossa experiência. BMC Cardiovasc Disord, v. 14, n. 106, p 1-6, ago. 2014.

HUANG, X. et al. Influenza infection and Kawasaki disease. Revista da Sociedade Brasileira de Medicina Tropical, v. 48, n. 3, p. 243-248, jun. 2015.

KAYIRAN, S. M.; DINDAR, A. N.; GURAKAN, B. An evaluation of children with Kawasaki disease in Istanbul: a retrospective follow-up study. Clinical Science, v. 65, n. 12, p. 1261-1265, dez. 2010.

KENTSIS, A. et al. Urine proteomics for discovery of improved diagnostic markers of Kawasaki disease. EMBO Mol Med, v. 5, n. 2, p. 210-220, fev. 2013.

KIM, J. S.; KWON, S. H. Atypical Kawasaki disease presenting a retropharyngeal abscess. Braz J Otorhinolaryngol, v. 82, n. 4, p. 484-486, 2016.

LIU, M. Y. et al. Risk factors and implications of progressive coronary dilatation in children with Kawasaki disease. BMC Pediatrics, v. 17, n. 139, p. 1-7, jun. 2017.

MAGALHÃES, C. M. E. Estudo da prevalência da perda auditiva neurossensorial como complicação da doença de Kawasaki. Tese (Doutorado em Ciências da Saúde) - Faculdade de ciências da saúde, Universidade Federal de Brasília, Brasília. 2008.

MAGALHÃES, C. M. R.; ALVES, N. R. M. Doença de Kawasaki. In: I. Burns, DAR, Campos Júnior D, Silva LR, Borges WG. Tratado de pediatria: Sociedade Brasileira de Pediatria. 4. ed. Barueri, SP: Manole, 2017. p.1825-1835.

RC: 82505

Disponível em: https://www.nucleodoconhecimento.com.br/salud/enfermedad-dekawasaki 
MARTÍNEZ, U. M.; MÉNDEZ, F. M. Manejo anestésico del paciente com enfermedad de Kawasaki durante la cirugía de revascularización coronaria: informe de um caso. Archivos de Cardiología de México, v. 83, n. 4, p. 267-272, abr. 2013.

MEHNDIRATTA, S. et al. A case of incomplete and refractory Kawasaki disease: Diagnostic and therapeutic challenges. The South African Journal of Child Health, v. 8, n. 1, p. 37-38, fev. 2014.

MUTLUER, F. O.; ÇELIKER, A. Comment on Ligature of the Left Main Coeonary Artery after Surgery in Kawasaki Disease: Case Report. Brazilian Journal of Cardiovascular Surgery, v. 34, n.3, p. 382, jul. 2019.

PHILIP, S. et al. Role of Antioxidants in Horse Serummediated Vasculitis in Swine: Potential Relevance to Early Treatment in Mitigation of Coronary Arteritis in Kawasaki Disease. Pediatrics and Neonatology, v. 58, n. 4, p. 328-337, fev. 2017.

PRINTZ, B. F. Noncoronary Cardiac Abnormalities Are Associated With Coronary Artery Dilation and With Laboratory Inflammatory Markers in Acute Kawasaki Disease. Journal of the American College of Cardiology, v. 57, n. 1, p. 68-92, jan. 2011.

RODRIGUES, M. et al. Doença de Kawasaki e Complicações Cardiovasculares em Pediatria. Birth and growth medical jornal, v.27, n. 1, p. 54-58, fev. 2017.

ROSSI, F. S. et al. Extensa linfadenite cervical mimetizando adenite bacteriana como primeira manifestação da doença de Kawasaki. Einstein, v. 13, n. 3, p. 426429, jul./set. 2015.

ROWLEY, A. H. et al. Allograft Inflammatory Factor-1 Links T-Cell Activation, Interferon Response, and Macrophage Activation in Chronic Kawasaki Disease Arteritis. Journal of the Pediatric Infectious Diseases Society, v. 6, n. 3, p. 94102, set. 2017.

RC: 82505

Disponível em: https://www.nucleodoconhecimento.com.br/salud/enfermedad-dekawasaki 
SCARDINA, G. A. et al. Oral necrotizing microvasculitis in a patient affected by Kawasaki disease. Medicina Oral Patologia Oral y Cirugia Bucal, v. 12, n. 8, p. 560-564, dez. 2007.

SOTELO, N.; GONZÁLEZ, L. A. Kawasaki disease: A rare pediatric pathology in Mexico Twenty cases report from the Hospital Infantil del Estado de Sonora. Archivos de Cardiologia de Mexico, v. 77, n. 4, p. 299-307, dez. 2007.

XIE, X. F. et al. Proteomics study of serum exosomes in Kawasaki disease patients with coronary artery aneurysms. Cardiology Journal, v. 26, n. 5, p. 584-593, fev. 2018.

YAP, C. Y.; LIN, L. H.; WANGI, N. K. An atypical presentation of Kawasaki disease: a 10- year-old boy with acute exudative tonsillitis and bilateral cervical lymphadenitis. CLINICS, v. 67, n. 6, p. 689-692, 2012.

Enviado: Abril de 2021.

Aprobado: Abril de 2021. 